\title{
EVALUACIÓN DEL FACTOR DE SEGURIDAD EN TALUDES DE TERRAPLENES CARRETEROS ALTOS ANTE CARGA SÍSMICA
}

\author{
Milena Mesa Lavista ${ }^{(1)}$, José Álvarez Pérez ${ }^{(1)}$ y Jorge H. Chávez Gómez ${ }^{(1)}$
}

\begin{abstract}
RESUMEN
Cuando se construye un terraplén carretero, una de las variables analizadas en el diseño es la obtención del factor de seguridad para determinar la estabilidad y seguridad de los taludes. Este diseño se realiza haciendo uso de un análisis estático y, en casos más específicos y aislados haciendo un análisis sísmico. En el presente trabajo se realiza un estudio del comportamiento del factor de seguridad en taludes de terraplenes carreteros altos frente a la solicitación sísmica. Para llevar a cabo el presente estudio se realizó un diseño de experimento $3^{2}$. En el diseño de experimento se varió la altura del terraplén en tres niveles y el nivel de sismicidad, construyendo acelerogramas a partir de los espectros de diseño considerando los parámetros de la sección 3.1 del Manual de diseño de obras civiles de la comisión federal de electricidad de México. En la obtención de los resultados se empleó el método de los elementos finitos (MEF) y el método de Newmark. Como herramienta computacional se empleó el software especializado Geo-Studio con el sub-módulo para el análisis dinámico QUAKE/W, y el sub-módulo para el análisis de estabilidad SLOPE/W. Los resultados obtenidos muestran la necesidad de revisar estas obras frente al sismo sobre todo en zonas de sismicidad alta cuando su altura supere los $12 \mathrm{~m}$.
\end{abstract}

Palabras clave: factor de seguridad; estabilidad de taludes; terraplenes altos; solicitación sísmica

\section{SAFETY FACTOR EVALUATION IN HIGH EMBANKMENT ROADS SLOPES CONSIDERING A SEISMIC LOAD}

\begin{abstract}
When a road embankment is built, the safety factor is analyzed as one of the most important variables in the design; since it is necessary that slopes are safe and stable. In most cases, a static analysis is used for design purposes. This paper presents results of a study on the seismic behavior of the safety factor of slopes, in high road embankments under seismic action. An experimental design 32 was performed for this study. In the experimental design, three different embankment heights were considered and the level of seismicity was varied through accelerograms from the design spectrum bearing in mind the parameter of the section 3.1 of the "Manual de diseño de obras civiles de la comisión federal de electricidad" of Mexico. To obtain the results, the finite element method (FEM) and the Newmark method were employed by the software Geo-Studio; using the sub-modulus

Artículo recibido el 10 de noviembre de 2017 y aprobado para su publicación el 27 de abril de 2020. Se aceptarán comentarios y/o discusiones hasta cinco meses después de su publicación.

(1) Facultad de Ingeniería civil, Universidad Autónoma de Nuevo León, Ave. Universidad S/N, Ciudad Universitaria, San Nicolás de los Garza, Nuevo León, México, C.P. 66451. milena.mesal@uanl.mx; jose.alvarezpe@uanl.mx; Jorge.chavezg@uanl.mx
\end{abstract}

DOI: $10.18867 /$ ris. 103.489 
QUAKE/W for dynamic analysis, and the sub-modulus SLOPE/W for stability analysis. The results shown the necessity to review these structures in high intensity seismic regions for embankments higher than $12 \mathrm{~m}$.

Keywords: safety factor; slope stability; high embankments; seismic load

\section{INTRODUCCIÓN}

Los análisis de estabilidad de los taludes pueden ser abordados con diferentes enfoques (Cuanalo et al., 2011; Álvarez et al., 2015; Mesa y Guada, 2013; NC-856:2011, 2011; Zenghui et al., 2014) y el problema consiste en determinar las pendientes máximas que permitan construirlos de una manera estable y segura, evitando las fallas por deslizamientos, vuelco, desprendimientos, etc. Evaluar la estabilidad de un talud implica un estudio en el cual se debe, entre otros factores: caracterizar la resistencia del suelo, establecer el perfil del talud, así como las condiciones de filtración y agua subterránea, seleccionar una superficie de deslizamiento o falla y calcular su factor de seguridad. A partir de este factor de seguridad (el cual se elige en base al destino del talud) se determina la superficie de falla crítica (Ortiz, 2014; Gallego et al., 2012).

Los métodos para evaluar la estabilidad de los taludes son diversos. Los de equilibrio límite (Fellenius, 1936; Bishop et al.; 1986, Janbú, 1996; Morgenstern N. , 1965; Spencer, 1967) están basados en el método de las dovelas, dividiendo la masa de suelo en dovelas o rebanadas. Un análisis de equilibrio límite permite obtener un factor de seguridad teniendo en cuenta los valores de la resistencia al cortante en el momento de la falla (ec. 1). Una vez que se han determinado las propiedades de la resistencia a corte de los suelos y las propiedades geométricas del talud, se puede proceder a calcular el factor de seguridad (FS) (Mesa y Guada, 2013).

$F S=\frac{\sum S_{r}}{\sum S_{m}}$

donde:

$S_{r}$ es la fuerza cortante resistente

$S_{m}$ son las fuerzas cortantes movilizantes a lo largo de toda la longitud de la superficie de falla

Tanto para los terraplenes carreteros como para los terraplenes de presas de tierra se define un FS mínimo que está en función de la importancia de la obra. En los terraplenes carreteros para realizar el análisis estáticos de forma global es usual tomar el valor de 1.5 como factor de seguridad mínimo para que se considere que el talud es estable y seguro y se garantice un tránsito seguro del flujo vehicular (Torres Vila, 1986; NC-856:2011, 2011; Mesa et al., 2014). Sin embargo, algunas normas y autores consideran un FS mínimo para terraplenes y presas en función del análisis realizado (estático y dinámico) (tabla 1) (AASHTOLRFD, 2014; DM-7.01, 1986; DM-7.02, 1987; FHWA-NHI-11-032, 2011; Alva e Infantes, 2001; CE-020, 2012; Abramson et al., 2002; Gazetas et al., 2014). 
Tabla 1. Factores de seguridad permisibles considerados por diferentes normas y autores según el tipo de análisis

\begin{tabular}{ccc}
\hline Referencia & Análisis estático & Análisis dinámico \\
\hline DM-7.01 (1986) y DM-7.02 (1987) & 1.5 & $1.2-1.15$ \\
Torres Vila (1986) & 1.5 & - \\
Alva e Infantes (2001) & - & $>1$ \\
Abramson et al. (2002) & - & $>1$ \\
FHWA-NHI-11-032(2011) & - & 1.1 \\
NC-856:2011 (2011) & 1.5 & - \\
CE-020 (2012) & 1.5 & 1.25 \\
AASHTO-LRFD (2014) & $1.33-1.53$ & 1.1 \\
\hline
\end{tabular}

Muchos autores realizan análisis pseudo-estáticos (ec. 2) y dinámicos para determinar la estabilidad de los taludes de presas (Ruesta et al., 2006; Álvarez et al., 2015; Hernández, 2012; Franke, 2010; Ortiz, 2014; Talukdar et al., 2016); siendo la minoría de autores los que dedican el estudio a terraplenes carreteros frente a eventos sísmicos (Suárez, 2008; Botero y Romo, 2006). En ese sentido en el presente trabajo se realiza un estudio del comportamiento del FS en terraplenes carreteros altos considerando un análisis dinámico.

$F S=\frac{c * L *\left[\left(W-F_{v}\right) * \cos \alpha-F_{h} \operatorname{sen} \alpha\right] \tan \varphi}{\left(W-F_{v}\right) * \operatorname{sen} \alpha+F_{h} \cos \alpha} ; \quad F_{h}=\frac{a_{h} * W}{g} ; \quad F_{v}=\frac{a_{v} * W}{g}$

donde:

$F_{h}, F_{v}$ son las fuerzas pseudo-estáticas actuantes, horizontal y vertical

$a_{h}, a_{v}$ son las aceleraciones máximas horizontal y vertical

$c$ es la cohesión

$L$ es la longitud del plano de falla

$\varphi$ es el ángulo de fricción

$W$ es el peso de la masa deslizante

Se define un terraplén carretero alto en función de la altura del terraplén, la capacidad portante del suelo del cimiento, y el suelo componente del terraplén según la clasificación de suelos AASTHO. La tabla 2 muestra la clasificación de terraplenes altos obtenidas a partir de un análisis estático para alturas de hasta $30 \mathrm{~m}$ (Mesa, 2017).

Los métodos que estiman la deformación de la superficie de falla bajo la acción sísmica son los de Newmark, (1965), Markdisi y Seed, (1978), Jansen, (1990) y Sarma (1979) (Ortiz, 2014). En el método de Newmark se calcula la aceleración de rotura (AR) en función de un FS estático y la geometría del talud (ec. 3). Cuando las aceleraciones de la onda sísmica exceden el valor de rotura, la superficie de falla se mueve en forma de bloque, el resto del tiempo permanece en reposo, pudiéndose calcular las deformaciones acumuladas durante todo el movimiento sísmico (Sepúlveda, 2011).

$A_{R}=(F S-1) \operatorname{sen} \beta$ 
Tabla 2. Clasificación de terraplenes altos de carretera a partir de un análisis estático (Mesa, 2017)

\begin{tabular}{|c|c|c|c|c|}
\hline \multirow{2}{*}{$\mathrm{H}_{\mathrm{T}}(\mathrm{m})$} & \multirow{2}{*}{$\begin{array}{l}\text { CBR in situ en la } \\
\text { cimentación del } \\
\text { terraplén }\end{array}$} & \multicolumn{3}{|c|}{ Clasificación de suelos AASHTO para terraplenes } \\
\hline & & A-1 & A-2 & A-3 \\
\hline$\leq 6$ & $\geq 3 \%$ & \multicolumn{2}{|l|}{$1.5: 1$} & $2: 1$ \\
\hline \multirow{2}{*}{$\leq 12 \mathrm{~m}$} & $3 \%$ & \multicolumn{3}{|c|}{ No utilizable } \\
\hline & $\geq 5 \%$ & \multicolumn{3}{|c|}{ 2:1-1.5:1 o $2: 1$-berma-1.5:1 } \\
\hline \multirow{3}{*}{$\leq 18 \mathrm{~m}$} & $3 \%$ & \multicolumn{3}{|c|}{ No utilizable } \\
\hline & $\geq 5 \%$ & \multicolumn{2}{|l|}{ 2:1-berma-2:1-1.5:1 } & \multirow{2}{*}{$2: 1$-berma- $2: 1$} \\
\hline & $\geq 15 \%$ & 1.5:1-berma-1.5:1-berma-1.5:1 & & \\
\hline \multirow{3}{*}{$\leq 24 \mathrm{~m}$} & $3 \%$ & \multicolumn{3}{|c|}{ No utilizable } \\
\hline & $\geq 5 \%$ & \multicolumn{3}{|c|}{ 2:1-berma-2:1-berma-2:1-berma-2:1 } \\
\hline & $\geq 15 \%$ & \multicolumn{2}{|l|}{$2: 1-1.5: 1$-berma-2:1-1.5:1 } & $\begin{array}{l}\text { 2:1-berma-2:1-berma- } \\
2: 1 \text {-berma- } 2: 1\end{array}$ \\
\hline \multirow{3}{*}{$\leq 30 \mathrm{~m}$} & $3 \%$ & \multicolumn{3}{|c|}{ No utilizable } \\
\hline & $\geq 5 \%$ & \multicolumn{3}{|c|}{ 2:1-berma-2:1-berma-2:1-berma-2:1-berma-2:1 } \\
\hline & $\geq 15 \%$ & $\begin{array}{c}2: 1 \text {-berma-2:1-1.5:1-berma- } 2: 1- \\
1.5: 1\end{array}$ & & 2:1-berma-2:1 \\
\hline
\end{tabular}

Con el desarrollo de los métodos numéricos en la resolución de las ecuaciones diferenciales, el método de los elementos finitos (MEF) (Zienkiewicz y Taylor, 2002; Oñate, 2009) permite obtener el FS de los taludes teniendo en cuenta un estado esfuerzo-deformacional del suelo más cercano a la manera de trabajo de estos elementos estructurales.

El software Geo-Studio es una herramienta que emplea el MEF y que tiene implementado el módulo de análisis dinámico QUAKE/W (GEO-SLOPE, 2008) y el módulo de análisis de estabilidad de taludes SLOPE/W (GEO-SLOPE, 2012).

El módulo QUAKE/W permite introducir la solicitación sísmica al suelo en coordenadas de las aceleraciones de la gravedad vs tiempo. La acción del sismo es aplicada como fuerzas inerciales, estas fuerzas hacen que los esfuerzos en el suelo oscilen, y se analiza cada intervalo de tiempo, obteniéndose los resultados parciales que afectan el estado esfuerzo-deformación. A lo largo de una superficie de deslizamiento potencial, la resistencia al corte movilizada disminuye y aumenta en respuesta a las fuerzas de inercia. Puede haber momentos durante la sacudida en que la resistencia al corte movilizada excede la resistencia al corte disponible, lo que provoca una pérdida temporal de estabilidad. Durante estos momentos cuando el factor de seguridad es menor que la unidad, el suelo puede experimentar cierto desplazamiento. Una acumulación de estos movimientos puede manifestarse como un desplazamiento permanente. Con las nuevas condiciones se analiza el modelo en el siguiente intervalo de tiempo, y así sucesivamente hasta que termina el registro sísmico.

Para obtener el estado esfuerzo-deformación a partir de la solicitación sísmica el software emplea el método propuesto por Ishibashi y Zhanh (Ishibashi y Zhang, 1993) para obtener el módulo de corte dinámico.

$G=K(\gamma) f(e) \bar{\sigma}_{0}^{m(\gamma)}$

donde:

$G$ : módulo de corte dinámico

$K(\gamma)$ : función decreciente cíclica de la deformación cortante en una amplitud muy pequeña $\gamma \leq 10^{-6}$ 
$f(e)$ : función de la relación de vacíos

$\bar{\sigma}_{0}$ : presión de confinamiento media efectiva

$m(\gamma)$ : incremento de la función de $\gamma$

Una vez obtenido los resultados esfuerzo-deformación generados durante la solicitación sísmica, se calcula el FS mediante la aplicación del módulo SLOPE/W, para cada intervalo de tiempo (ec. 1). El módulo SLOPE/W permite realizar el cálculo del factor de seguridad en el talud empleando: a) el método de elementos finitos, donde se obtiene el comportamiento del FS para cada aceleración del terreno y el FS final y; b) el método de Newmark (bloques deslizantes), que brinda el FS último resultante de la deformación permanente del talud. En este caso se emplea el método de las dovelas.

Desarrollando el modelo numérico en estos dos módulos previamente mencionados, es posible determinar el FS de taludes carreteros al aplicar al suelo una solicitación sísmica. En el presente trabajo, el objetivo principal es analizar el comportamiento del FS frente al sismo, de terraplenes carreteros altos. Para llevar a cabo el objetivo, se realizó un diseño de experimento 32, variando dos factores (acelerograma y altura del terraplén) en tres niveles, posteriormente se realizan las simulaciones correspondientes a cada modelo, obteniendo los resultados que se muestran en la sección 3. Finalmente se brindan las conclusiones en la sección 4.

\section{MATERIALES Y MÉTODOS}

\section{Definición de las variables independientes}

Teniendo en cuenta la clasificación de terraplenes carreteros altos (tabla 2) (Mesa, 2017); y las categorías de sismicidad en donde se clasifican los sitios para presas en el Manual de diseño de obras civiles de México (MDOC, 2008) (tabla 3), se realizó un diseño de experimento 32 para determinar el comportamiento del FS frente a la solicitación sísmica. Los factores tenidos en cuenta fueron: (1) acelerogramas (variados en tres niveles de intensidad), obtenidos a partir de tres espectros de diseño teniendo en cuenta una sismicidad baja, media y alta y (2) la altura del terraplén (variado en tres niveles) (figura 1). A partir del diseño de experimento definido se realizaron los modelos numéricos para un total de 9 simulaciones numéricas.

Tabla 3. Clasificación de sitios para presas según la sismicidad [tomado de (MDOC, 2008)]

\begin{tabular}{cc}
\hline Sismicidad & Aceleración máxima del terreno $\left(\mathrm{a}_{0}\right)$ en $\mathrm{g}$ \\
\hline Baja & $<0.1$ \\
Media & $0.1 \leq \mathrm{a}_{0} \leq 0.4$ \\
Alta & $>0.4$
\end{tabular}

\begin{tabular}{|c|c|c|c|}
\hline \multirow{5}{*}{$\begin{array}{c}3^{2} \\
2 \text { factores } \\
3 \text { niveles }\end{array}$} & Sismicidad & & Altura del terraplén \\
\hline & Baja & & $6 \mathrm{~m}$ con talud $1.5: 1$ \\
\hline & & & $12 \mathrm{~m}$ con talud \\
\hline & Media & $2 \pi$ & $2: 1-1.5: 1$ \\
\hline & Alta & עי & $\begin{array}{c}18 \mathrm{~m} \text { con talud } \\
2: 1-\text { berma }-2: 1-1.5: 1\end{array}$ \\
\hline
\end{tabular}

Figura 1. Diseño de experimento 


\section{Generación de sismos sintéticos}

Para la generación de los sismos sintéticos se consideraron tres zonas de sismicidad según el criterio de la tabla 3, agrupando las zonas A y B de la figura 2 en zonas de sismicidad baja, la $\mathrm{C}$ en zona de sismicidad media y la $\mathrm{D}$ en zona de sismicidad alta. A partir de un enfoque probabilístico y teniendo en cuenta que las variables a definir son numerosas, se determinó, en un primer nivel del problema, acotar algunas variables deterministamente. Primeramente, se ha supuesto que, como lo especifica la norma (MDOC, 2008) el peso volumétrico del suelo es homogéneo y que, con el amortiguamiento de 5\% se tiene una razonable idealización de los suelos que suelen encontrarse en la práctica profesional. El tiempo de duración del sismo sintético se definió de 10 segundos, pues se obtuvieron resultados de sismos ocurridos provenientes del SAC (Christopher M et al., 2007; Malley, 1998; SAC, 1999).

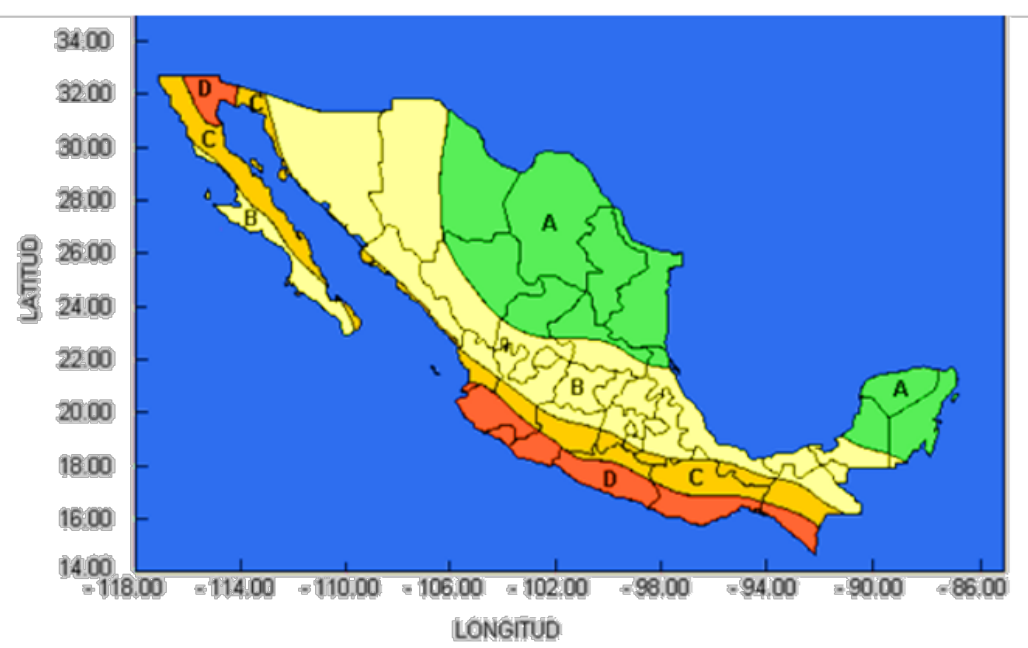

Figura 2. División de zonas sísmicas en México según el Manual de Diseño de Obras Civiles de 1993 [tomado de (Clemente, 2010; MDOC, 2008)]

SAC es una empresa conjunta de la Asociación de Ingenieros Estructurales de California (SEAOC), el Consejo de Tecnología Aplicada (ATC) y las Universidades de California para la Investigación en Ingeniería de Terremotos (CUREe), formada específicamente para abordar las necesidades tanto inmediatas como a largo plazo relacionadas con la resolución del problema en ingeniería sísmica (SAC, 1999; Malley, 1998).

Fueron analizados 10 sismos reales provenientes de la página del SAC (SAC, 1999) (figura 3). En el análisis de estos sismos, en el $90 \%$ de ellos, el tiempo de duración de las máximas aceleraciones se encuentra en el intervalo de 10 segundos. Además, considerando el tiempo de cómputo, se determinó que, para una primera evaluación de este fenómeno en terraplenes carreteros altos, se cumplía el objetivo del trabajo. La estructura se clasificó en el "Grupo B” según el MDOC-2008.

Para la generación de los acelerogramas se realizó un total de 50 sismos sintéticos para cada zona de sismicidad (baja, media y alta) con la ayuda del software SIMQKE (Gelfi, 2012) teniendo en cuenta los espectros de diseño que respondieran a las tres zonas de sismicidad antes mencionadas; según los parámetros del epígrafe 3.1 del MDOC-2008 y la aceleración máxima del terreno correspondientes a la tabla 3. Una vez obtenidos los sismos sintéticos para cada zona, se realizó un trabajo estadístico donde se obtuvo un acelerograma que correspondiese a una media representativa de los 50 resultados. En la figura 4 se muestran los acelerogramas introducidos en el software especializado Geo-Studio, para la simulación de la solicitación sísmica. 

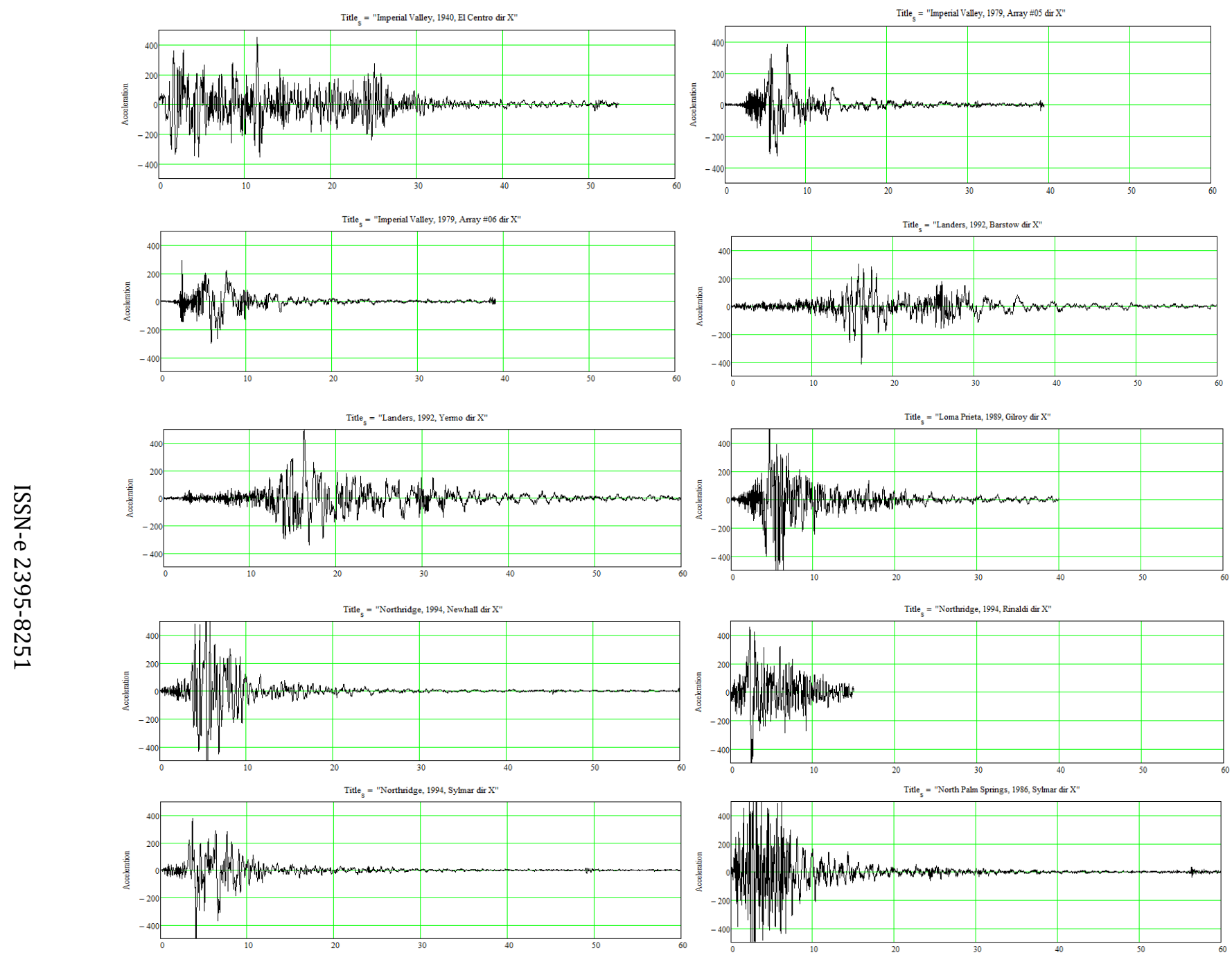

Figura 3. Sismos obtenidos del SAC 

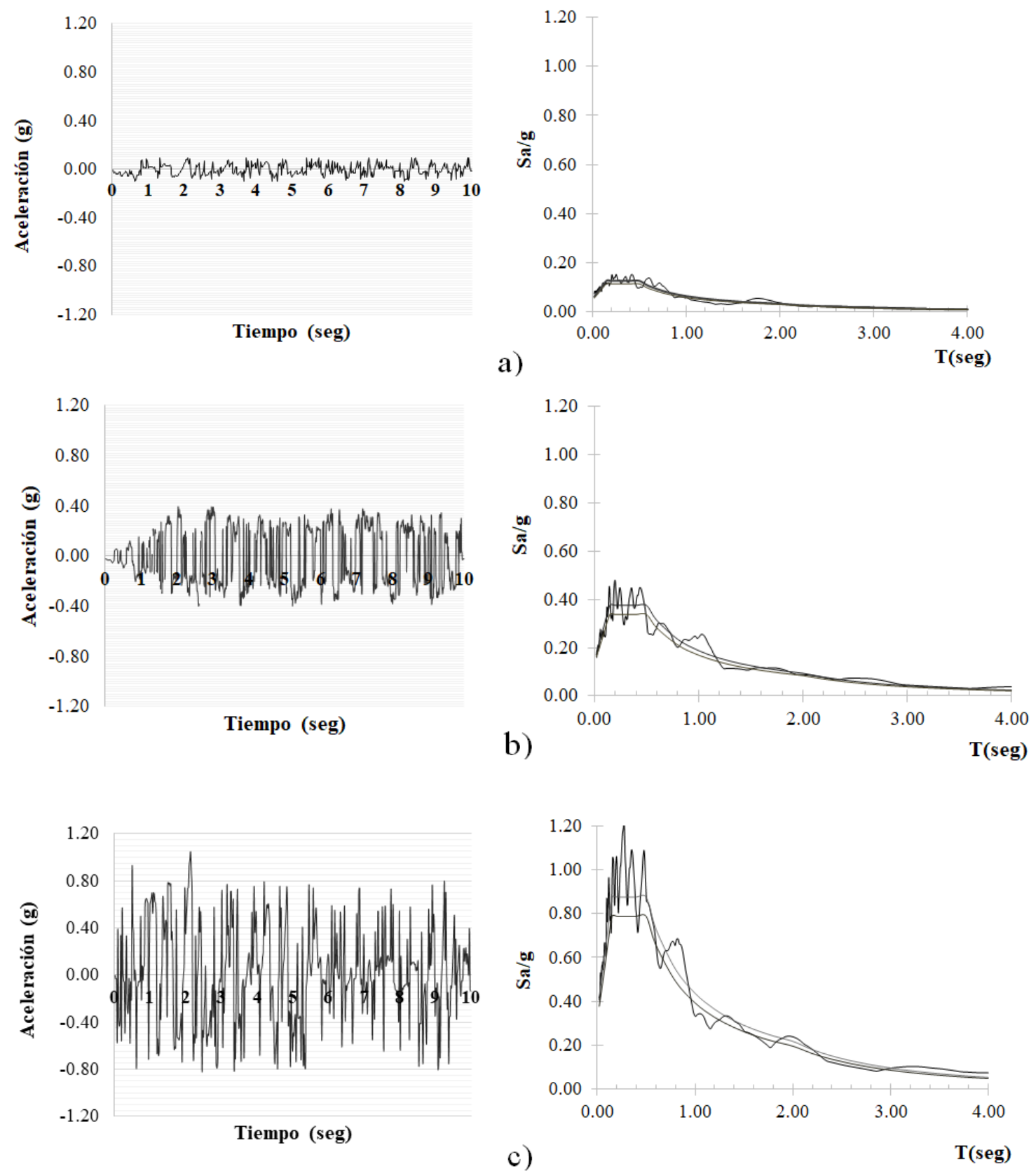

Figura 4. Acelerogramas y espectros obtenidos para las simulaciones: a) zona de sismicidad baja, b) zona de sismicidad media, c) zona de sismicidad alta

\section{Confección de los modelos numéricos}

Para la confección de los modelos numéricos en las diferentes alturas se tuvo en cuenta un modelo matemático con una discretización del dominio de $50 \mathrm{~cm}$ y el empleo de los tipos de elementos finitos cuadriláteros de 4 nodos, y triangulares de 3 nodos a partir de trabajos precedentes de calibración matemática de manera simultánea (figura 5) (Mesa y Álvarez, 2011). El espaciamiento de la malla para este tipo de problemas, fue definido en $50 \mathrm{~cm}$, ya que con valores de malla más refinada se genera mayor cantidad de elementos finitos y los errores obtenidos (empleando las normativas de errores) no son significativos en comparación con el espaciamiento de la malla de $50 \mathrm{~cm}$ (ver figura 5) (Mesa y Álvarez, 2011). El tipo de 
mallado empleado fue estructurado, de forma tal que en las zonas de los taludes se emplearan elementos triangulares de tres nodos, y en el resto elementos cuadriláteros de cuatro nodos. La cantidad de nodos también fue determinada en el proceso de calibración matemática simultánea, a través de las normativas de errores (error absoluto, porcentaje de error absoluto, error medio cuadrático, error según la norma del espacio euclidiano y desviación media absoluta) (figura 5) (Mesa y Álvarez, 2011).

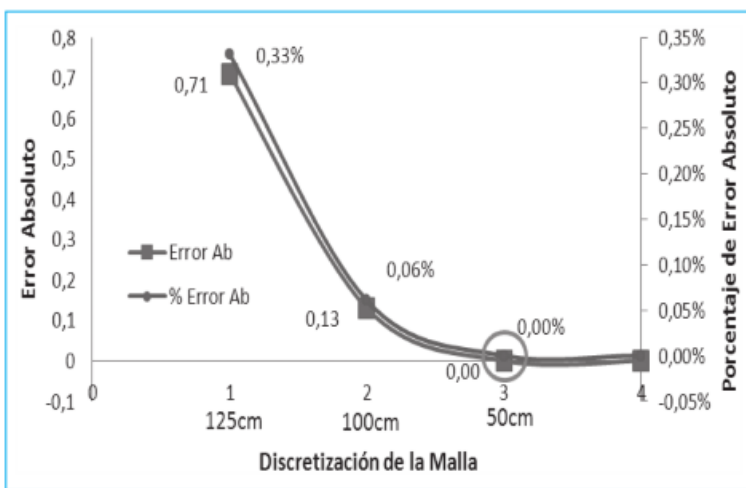

a)

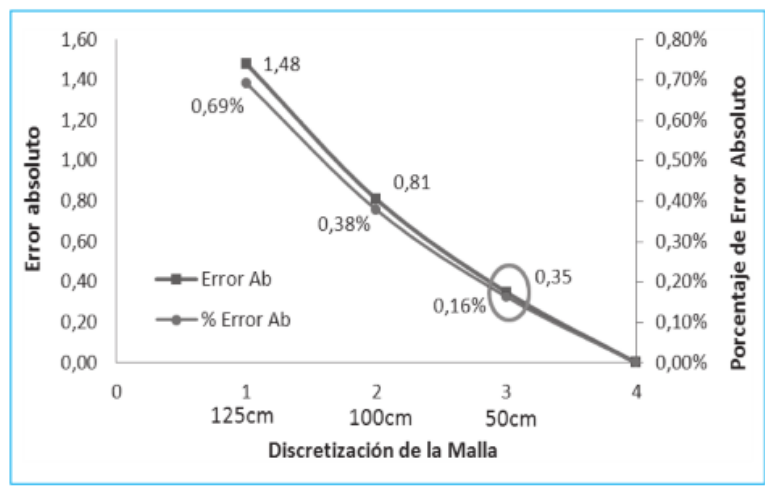

b)

Figura 5. Gráficos de la calibración matemática simultánea para a) Tipos de elementos finitos cuadrilátero de 4 nodos, b) Tipos de elementos finitos triangulares de 3 nodos. Tomado de (Mesa y Álvarez, 2011).

La concepción del modelo numérico (MEF) fue deformacional plano; ya que en un primer nivel del problema es común el empleo de las hipótesis del estado deformacional plano en la modelación de presas y terraplenes de carretas en los análisis estáticos. En ese sentido la concepción de un modelo tridimensional generaría mayor cantidad de grados de libertad y análisis adicionales en la calibración matemática.

Para la concepción del modelo numérico se tuvo en cuenta:

(1) Modelo geométrico: con profundidad de la potencia activa con un espesor equivalente de $45 \mathrm{~m}$, como indica el MDOC-2008 y dimensiones del subdominio $C=8 \frac{B_{T}}{H_{T}}$ (figura 6) (Mesa et al., 2016).

(2) Condiciones de frontera e interacciones: las condiciones de frontera se establecieron con ligaduras lineales permitiendo los desplazamientos horizontales, la interacción entre el suelo del cimiento y el suelo del terraplén está definida del tipo "tie", el cual es un contacto de unión del tipo cinemático.

(3) Modelo constitutivo: el modelo empleado fue el elasto-plástico no lineal de Mohr Coulomb en condiciones no drenadas, ya que, a pesar que los suelos considerados son granulares, la velocidad de aplicación de la carga para este caso es de corta duración, las propiedades empleadas de los suelos para el terraplén fueron obtenidas a partir de un análisis de las propiedades resistentes del suelo A-1 de la clasificación AASHTO (tabla 4) tomando como ángulo de dilatancia el $15 \%$ del ángulo de fricción (Simancas, 1999) y las propiedades del suelo del cimiento empleadas fueron obtenidas a partir de fijar y homogenizar un suelo con características de un $\mathrm{CBR}=15 \%$ (tabla 5), no fue considerado nivel freático.

(4) Modelo de carga: la carga fue introducida en los modelos a partir de los acelerogramas obtenidos, la fuerza actúa en cada nodo de la frontera en la base del modelo, estas fuerzas nodales se propagan hacia arriba provocando que cambie el estado esfuerzo-deformación del conjunto debido a las fuerzas inerciales. 


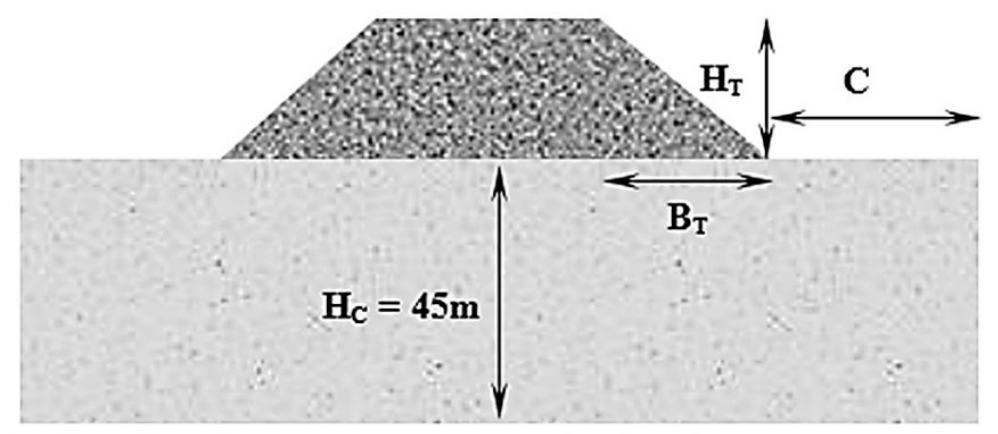

Figura 6. Relaciones geométricas empleadas para los modelos numéricos

Tabla 4. Parámetros físico-mecánicos del suelo A-1 en el terraplén (Ábalo, 1986; AASHTO, 2016)

\begin{tabular}{lc} 
Peso específico, $\gamma\left(\mathrm{kN} / \mathrm{m}^{3}\right)$ & 22.00 \\
Humedad, $w(\%)$ & 11.00 \\
Módulo de deformación, E $(\mathrm{MPa})$ & 40.00 \\
Cohesión, $C(\mathrm{kPa})$ & 2.00 \\
Ángulo de fricción, $\varnothing\left({ }^{\circ}\right)$ & 38.00 \\
Poisson, $\mu$ & 0.25 \\
Ángulo de dilatancia, $\Theta\left({ }^{\circ}\right)$ & 5.70 \\
\hline
\end{tabular}

Tabla 5. Propiedades físico-mecánicas del suelo del cimiento

Módulo de

Capacidad

portante CBR

$(\%)$ deformación

$E_{d}=$

$6.5 C B R^{0.65}$

$(\mathrm{kPa})$

$15 \%$

$\begin{array}{cccc}\text { Peso } & \text { Poisson } & \text { Cohesión } & \begin{array}{c}\text { Ángulo de } \\ \text { específico }\end{array} \\ \gamma\left(\mathrm{kN} / \mathrm{m}^{3}\right) & \mu & C(\mathrm{kPa}) & \varnothing\left(^{\circ}\right)\end{array}$

Ángulo de dilatancia $\Theta\left({ }^{\circ}\right)$ $=0.15 \varnothing\left(^{\circ}\right)$

\begin{tabular}{lllllll}
\hline $15 \%$ & 38000 & 22.00 & 0.30 & 10 & 40 & 6 \\
\hline
\end{tabular}

Los modelos geométricos concebidos finalmente para las simulaciones sísmicas se muestran en la figura 7 para el modelo de $6 \mathrm{~m}$ de altura, figura 8 para el de $12 \mathrm{~m}$ y figura 9 para el de $18 \mathrm{~m}$.

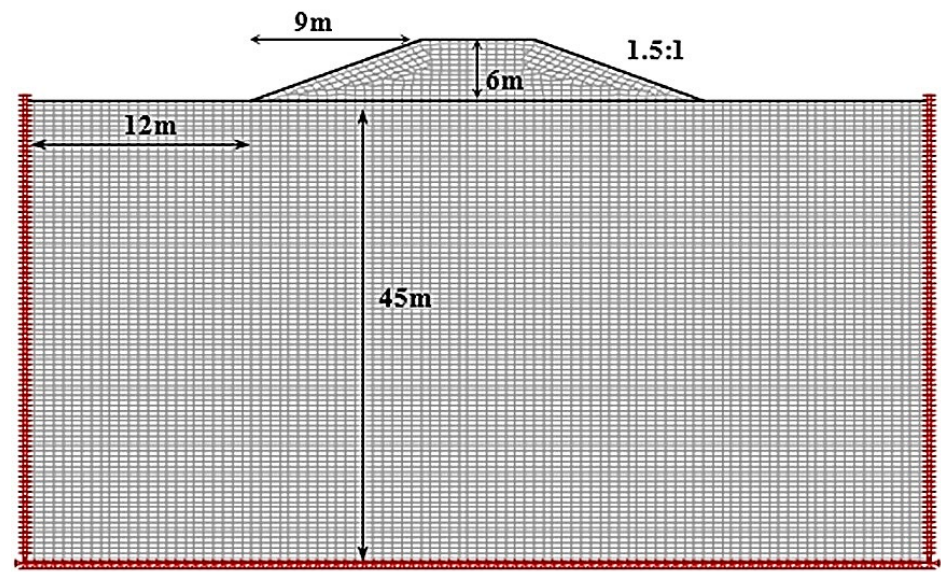

Figura 7. Modelo geométrico para el terraplén de $6 \mathrm{~m}$ de altura 


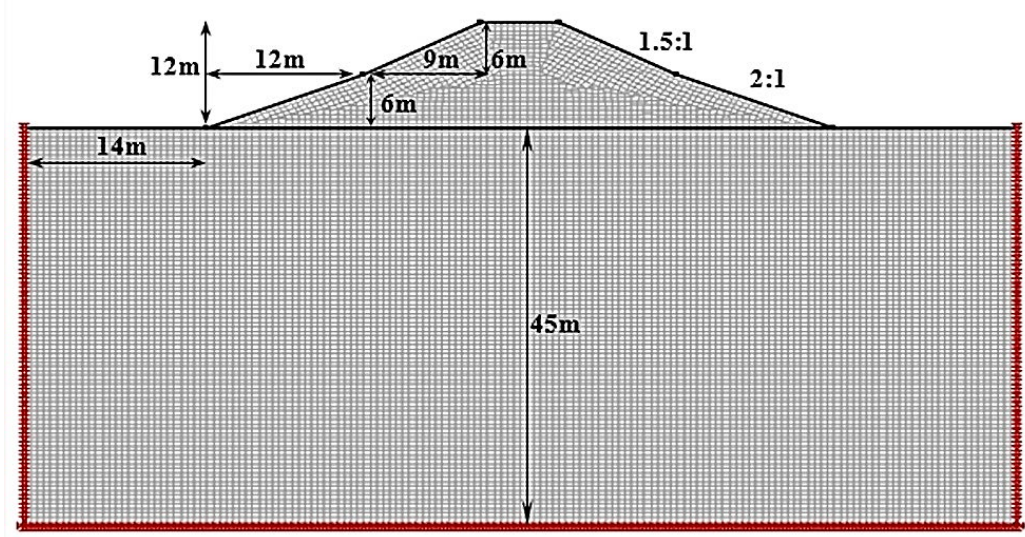

Figura 8. Modelo geométrico para el terraplén de $12 \mathrm{~m}$ de altura

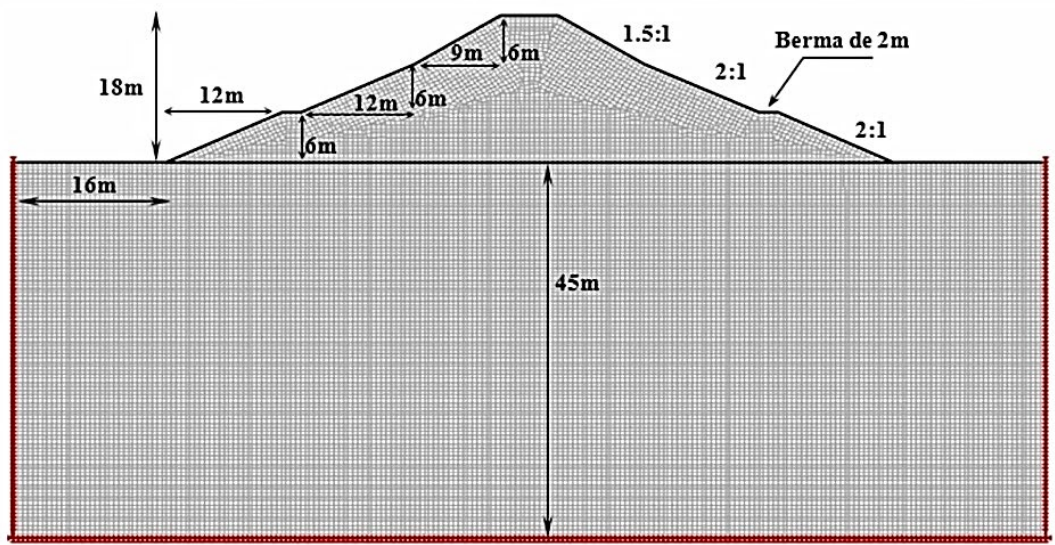

Figura 9. Modelo geométrico para el terraplén de $18 \mathrm{~m}$ de altura

De forma general, para la modelación y simulación de los sismos sintéticos en los terraplenes se empleó la estrategia de modelación que se presenta en la figura 10.

\section{RESULTADOS Y DISCUSIÓN}

Luego de las simulaciones realizadas se obtuvieron los resultados del comportamiento del FS durante la aplicación de los sismos sintéticos. Los resultados que se muestran en las figuras 12 a 16, representan el FS obtenido en cada instante de tiempo (paso de integración) para cada aceleración promedio del terreno en ese instante (acelerograma). Es decir, obtención del estado esfuerzo-deformación debido al vector desplazamiento resultante nodal producto de las fuerzas inerciales, y luego obtención del FS por elementos finitos y por deformaciones permanentes (Método de Newmark).

En las figuras 12 a 14, se muestran los resultados del modelo numérico de $6 \mathrm{~m}$ de altura teniendo en cuenta los tres acelerogramas simulados. La aceleración promedio que se muestra en las figuras es la transmitida al terraplén en el momento del sismo. El comportamiento del FS es el calculado mediante el método de Newmark para cada instante de aceleración en el terraplén. Los resultados que se muestran son del talud derecho, de ahí que, para aceleraciones promedio negativas en el terraplén el FS aumente y para aceleraciones promedio positivas en el terraplén el FS disminuya (debido al convenio de signos) (figura 11). 


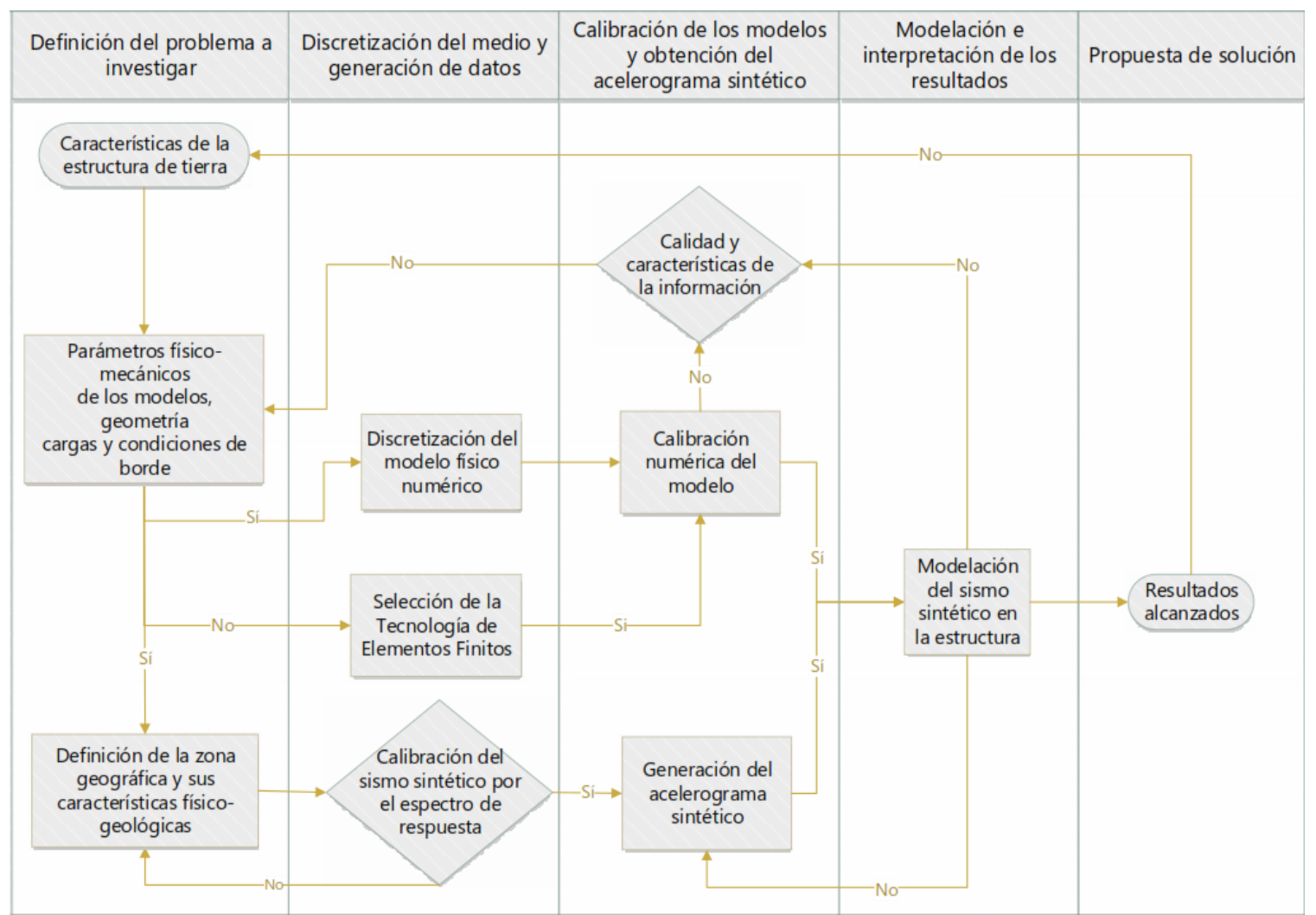

Figura 10. Estrategia de modelación empleada en el presente estudio. Tomado de (González et al., 2018)
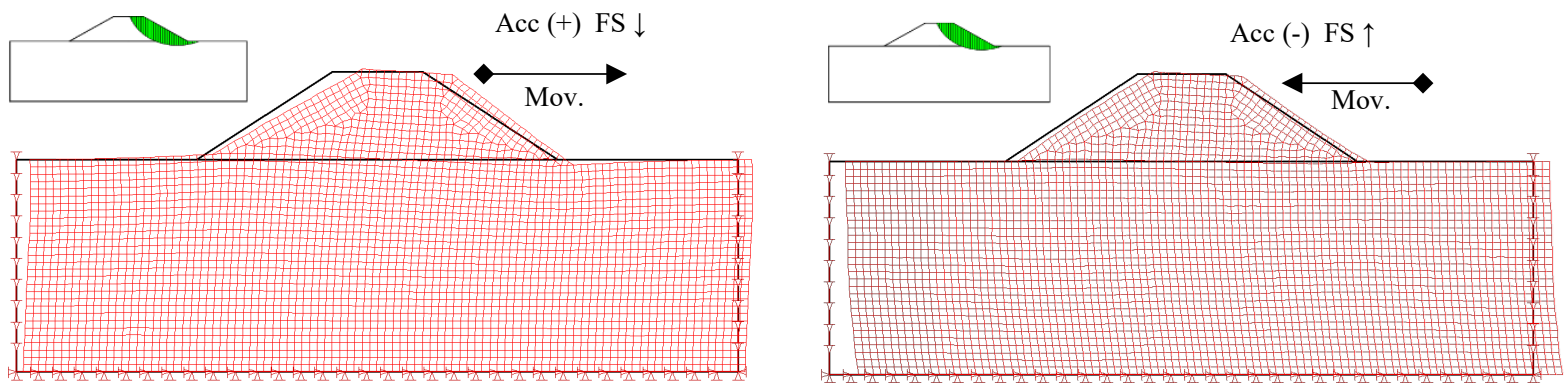

Figura 11. Deformada del terraplén en dos pasos de integración y convenio de signos para el ejemplo de 6 $\mathrm{m}$ de altura

Teniendo en cuenta que para los análisis estáticos el FS debe ser mayor a 1.5, y considerando los requisitos de la tabla 1 se siguió el criterio que luego de la ocurrencia del sismo, el FS debe ser mayor que 1 (Alva e Infantes, 2001). Sin embargo, durante la ocurrencia del evento sísmico este FS puede ser menor que 1, como lo plantea (Gazetas et al., 2014), ya que pueden surgir deformaciones permanentes en el suelo y esto no llevar a la falla del talud.

En las figuras 13 y 14, se observa que para valores de aceleraciones inferiores a $-0.20 \mathrm{~m} / \mathrm{s}^{2}$ los valores del FS fueron inferiores al FS mínimo para el análisis estático (1.5). Sin embargo, para los terraplenes de 12 m y $18 \mathrm{~m}$, localizados en zonas de sismicidad alta (figura 15 y 16), el valor del FS llegó a cero cuando la aceleración promedio en el terraplén alcanzó valores iguales o inferiores a $-1.00 \mathrm{~m} / \mathrm{s}^{2}$. De acuerdo a lo planteado por (Gazetas et al., 2014), este comportamiento durante el evento sísmico está permitido, pues se producen deformaciones permanentes que no implican la falla del talud. Sin embargo, una vez que concluye 
la solicitación sísmica, y el suelo del terraplén está permanentemente deformado, es necesario calcular el FS final y evaluar si es estable o no para la circulación del tráfico. En ese sentido se obtuvo un FS por el MEF y por el método de Newmark, y fue comparado con un FS permisible de 1 (Alva y Infantes, 2001), pues debe cumplirse que, al menos las fuerzas que provocan el deslizamiento sean iguales a las resistentes al final del sismo con las deformaciones permanentes alcanzadas.

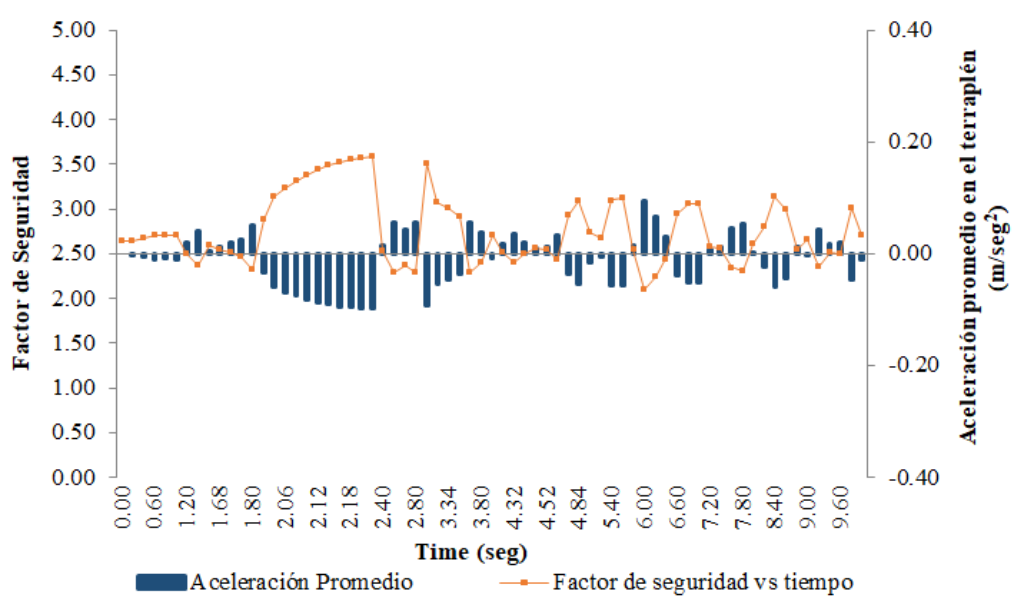

Figura 12. Comportamiento del factor de seguridad vs aceleración promedio transmitida al terraplén de 6 $\mathrm{m}$ de altura para una zona de sismicidad baja

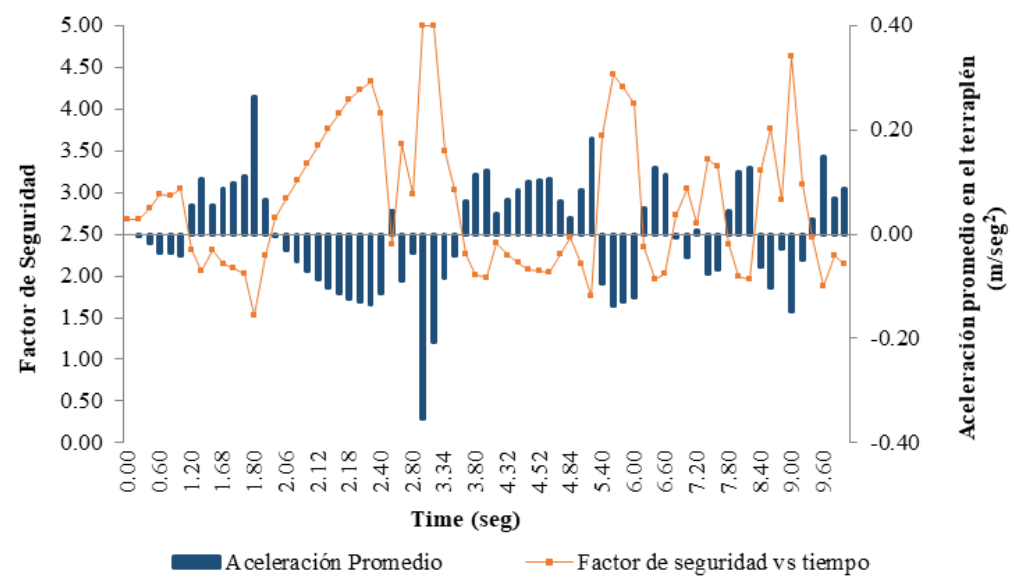

Figura 13. Comportamiento del factor de seguridad vs aceleración promedio transmitida al terraplén de 6 $\mathrm{m}$ de altura para una zona de sismicidad media 


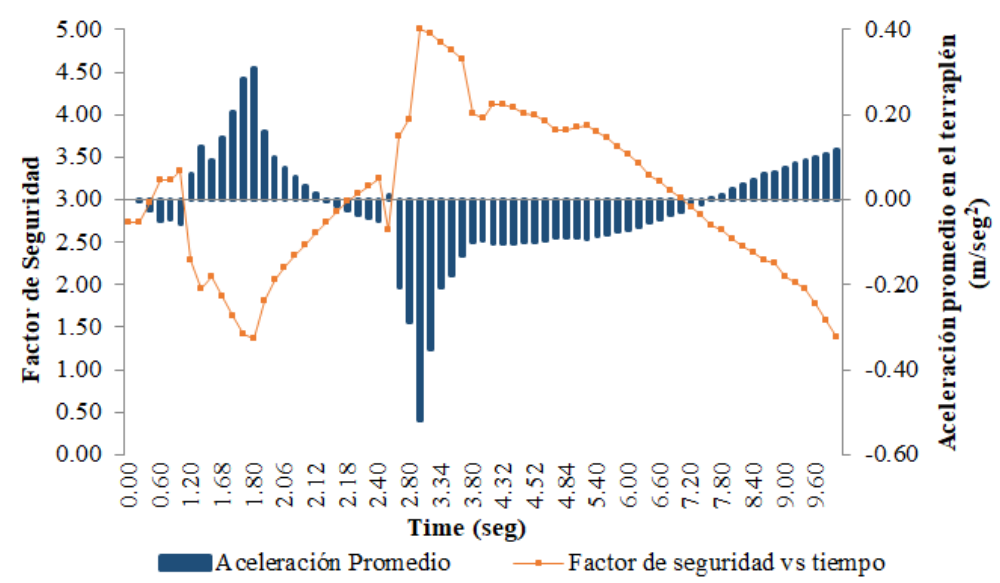

Figura 14. Comportamiento del factor de seguridad vs aceleración promedio transmitida al terraplén de 6 $\mathrm{m}$ de altura para una zona de sismicidad alta

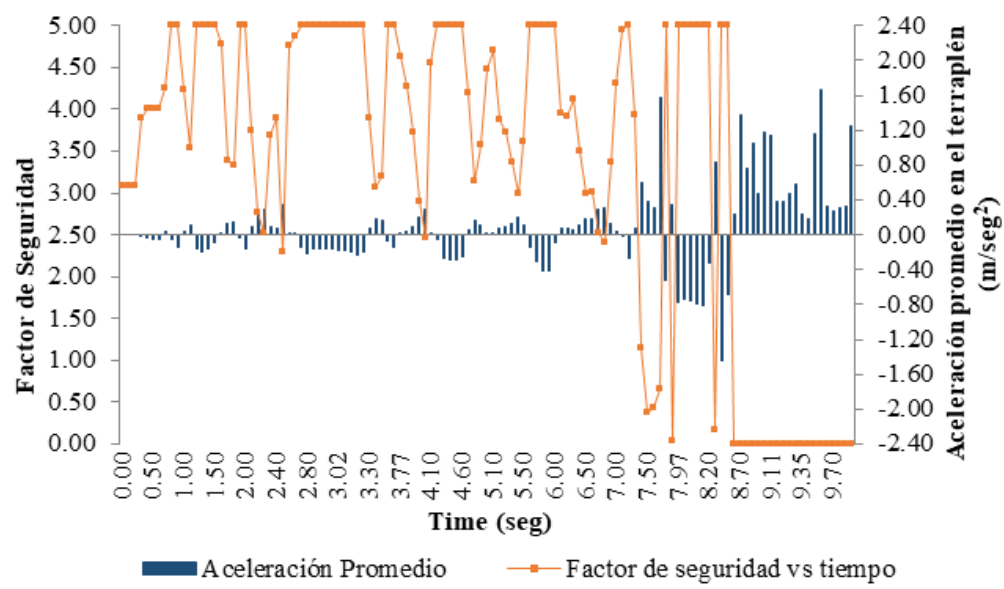

Figura 15. Comportamiento del factor de seguridad vs aceleración promedio transmitida al terraplén de 12 $\mathrm{m}$ de altura para una zona de sismicidad alta

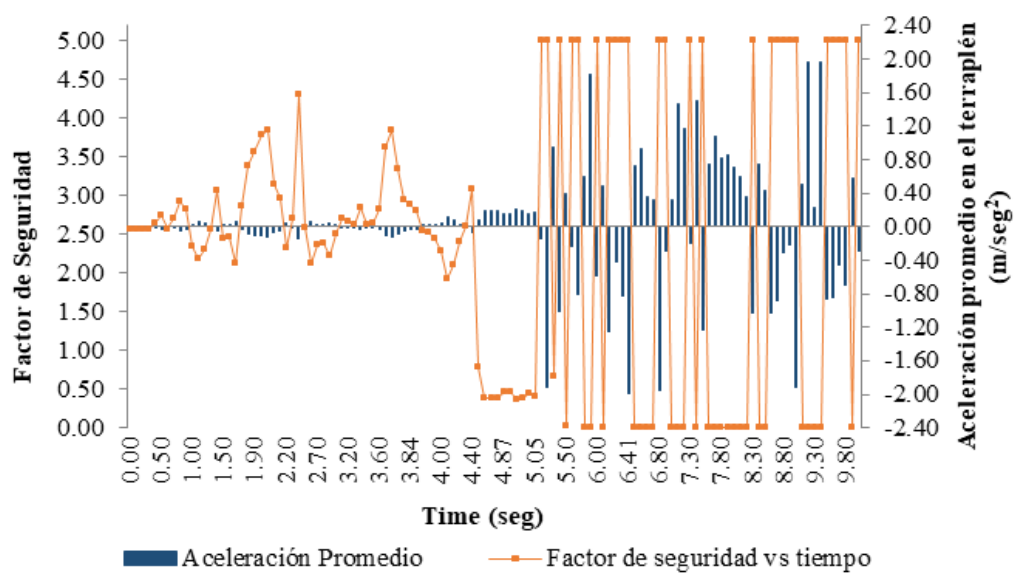

Figura 16. Comportamiento del factor de seguridad vs aceleración promedio transmitida al terraplén de 18 $\mathrm{m}$ de altura para una zona de sismicidad alta 
De forma general se puede observar en la tabla 6 que, luego de la acción sísmica y teniendo en cuenta las deformaciones permanentes alcanzadas, en los modelos simulados, el FS final fue mayor que 1 para las zonas de baja y mediana sismicidad, sin embargo, para los terraplenes de $12 \mathrm{~m}$ y $18 \mathrm{~m}$ para las zonas de sismicidad alta, el FS fue inferior a 1, tanto por el MEF como por el método de Newmark. Esto implica que para los casos analizados (12 m y $18 \mathrm{~m}$ ) existe un deslizamiento del talud para zonas de alta sismicidad $(\mathrm{FS}<1)$. Por lo tanto, se sugiere que, cuando los terraplenes sobrepasen los $12 \mathrm{~m}$ de altura, y la zona sea de sismicidad media-alta, se realice una revisión debido a la solicitación sísmica.

En el presente estudio, se empleó un tiempo de 10 segundos (como variable determinista) teniendo en cuenta que, en el 90\% de los sismos analizados del estudio del SAC (SAC, 1999) la ocurrencia de las aceleraciones máximas es en ese intervalo de tiempo. Sin embargo, en futuros trabajos se deberá abordar el estudio con mayores intervalos de tiempos de duración de estás aceleraciones máximas, ya que, debido a la intensidad y a la geometría de los taludes, los resultados finales del FS con las deformaciones permanentes alcanzadas será diferente. También es de interés la revisión del talud izquierdo, ya que, en estudios precedentes (caso de estudio de un terraplén de carretera de $7 \mathrm{~m}$ de altura) (González et al., 2018), se obtuvieron resultados diferentes de la variación del FS en el análisis del talud derecho e izquierdo, así como también se obtuvieron valores diferentes en la variación del FS al variar el amortiguamiento para un caso de estudio de una presa de tierra homogénea (González et al., 2018)

Tabla 6. Resultados del FS para los terraplenes analizados mediante el MEF y el método de Newmark

\begin{tabular}{ccccc}
\hline Método & Sismicidad & Terraplén de $6 \mathrm{~m}$ & Terraplén de $12 \mathrm{~m}$ & Terraplén de $18 \mathrm{~m}$ \\
\hline MEF & \multirow{2}{*}{ Baja } & 2.546 & 2.261 & 2.129 \\
Newmark & \multirow{2}{*}{ Nedia } & 1.392 & 2.040 & 2.857 \\
\hline MEF & \multirow{2}{*}{ Media } & 1.657 & 1.938 & 1.754 \\
Newmark & & 1.366 & 1.865 & 1.632 \\
\hline MEF & \multirow{2}{*}{ Alta } & 1.290 & $\mathbf{0 . 5 0 5}$ & $\mathbf{0 . 1 5 7}$ \\
Newmark & & & $\mathbf{0 . 0 0 4}$ & $\mathbf{0 . 0 0 0}$ \\
\hline
\end{tabular}

\section{CONCLUSIONES}

Una vez concluido el estudio de la evaluación del factor de seguridad de los terraplenes carreteros altos frente a la solicitación sísmica se llegó a las siguientes conclusiones:

- Durante el evento sísmico, estará permitido que el FS baje de 1, ya que pueden presentarse deformaciones permanentes y finalmente ser estable el talud luego de la ocurrencia de la acción sísmica (Gazetas et al., 2014).

- Cuando el evento sísmico acabe, deberá revisarse el FS final debido a las deformaciones permanentes alcanzadas en el terraplén, este deberá ser mayor o igual a 1 (Alva y Infantes, 2001).

- Cuando se proyecten terraplenes de carretera superiores a los $12 \mathrm{~m}$ de altura, se deberá valorar la sismicidad de la zona (media-alta) y realizar una revisión por concepto de solicitación sísmica.

- Para zonas de sismicidad baja y media es necesario realizar simulaciones con tiempos superiores a los $10 \mathrm{~s}$ y diferentes valores de amortiguamiento, ya que el comportamiento del FS podría variar para ambos casos (González et al., 2018)

En próximos trabajos se realizarán diseños de experimento más generalizadores donde se modelen sismos con un intervalo de tiempo mayor de $10 \mathrm{~s}$, otras alturas de terraplenes y estratificación en el suelo del terraplén con el objetivo de poder acotar en términos de varias superficies de respuestas el alcance de los enfoques estáticos y cuasi-estáticos para este tipo de estructuras hiperestáticas continuas 


\section{REFERENCIAS}

AASHTO-LRFD (2014). American association of state highway and transportation officials - Load and Resistance Factor Desing. En: SPECIFICATIONS, B. D. (ed.). Washington.

AASHTO (2016). Road standard. Section 207. Embankment.

Ábalo, M A (1986). Características de los suelos. En: ISPJAE (ed.). Ciudad de la Habana.

Abramson, L W, T S Lee, S Sharma y G M Boyce (2002), Slope stability and stabilization methods. J.Wiley \& Sons.

Alva, J E e M Infantes (2001), Diseño sísmico de presas de tierra y enrocado. En: Desastres, C. P. J. D. I. S. Y. M. D. (ed.) Curso Actualización profesional de dinámica de suelos. Lima, Perú.

Álvarez, M, J E Martínez y L Álvarez (2015), "Simulación físico-numérica para evaluar el comportamiento sismo resistente en presas de tierra". Memorias del XII Congreso Cubano de Informática y Geociencias (GEOINFO). Modelación matemática y Software aplicados a la Explotación Minera,

Bishop, C S, D W Armour y T C Hopkins (1986), "Design of highway embankments on unstable natural slopes". Research Report UKTRP-86-22. University of Kentucky.

Botero, E y M P Romo (2006), "Modelo bidimensional no lineal para el análisis del comportamiento dinámico de estructuras térreas". Revista de Ingeniería Sísmica, 1-32, DOI: 10.18867/RIS.74.68

CE-020 (2012), Estabilización de suelos y taludes. Ministerio de Vivienda, Contrucción y Saneamiento de Perú. Lima.

Clemente, A (2010), "Ley de atenuación de aceleración (PGA) y escalamiento de forma espectral sísmica para Querétaro, deducidos por análisis de trayectoria: Aplicada a Guerrero-Querétaro". Tesis de Maestría, Centro Universitario Querétaro, Qro.

Cuanalo, O A, A O Oliva y R Gallardo (2011), "Inestabilidad de laderas. Influencia de la actividad humana". Elementos, 84, 39-46

Christopher, M F, P Shahram y A Arzhang (2007), "Probabilistic performance-based optimal design of steel moment-resisting frames. I: Formulation". Journal of structural engineering (ASCE), Vol. 133, Issue 6, DOI: 10.1061/(ASCE)0733-9445(2007)133:6(757)

DM-7.01 (1986), Soil Mechanics. Design Manual 7.01. En: COMMAND, N. F. E. (ed.).

DM-7.02 (1987), Foundations \& Earth Structures. En: TRANSMITTAL, N. F. E. C. P. (ed.). Alexandria, Virginia.

Fellenius, W (1936), "Calculation of The Stability of Earth Dams", Second Congress on Large Dams. Washington D.C.

FHWA-NHI-11-032 (2011), Federal highway administration. LRFD Seismic analysis and design of transportation geotechnical features and structural foundations. Washington.

Franke, G (2010), "Efectos del sismo del 27 de febrero en presas de la región Comahue". VI Congreso argentino de presas y aprovechamientos hidroeléctricos,

Gallego, G, J C Leal, E Rojas y R S Mora (2012), "Efecto de la succión en la estabilidad de un talud de arena limosa". Ingeniería Investigación y Tecnología, XIII, 271-281

Gazetas G, I Anastasopoulos y E Garini (2014), "Geotechnical design with apparent seismic safety factors well-bellow 1". Soil Dynamics and Earthquake Engineering, 57, 37-45. DOI: 10.1016/j.soildyn.2013.10.002

GELFI, P. (2012), SIMQKE_GR. Versión-2.7 ed. Italia: University-of-Brescia.

GEO-SLOPE (2008), Dynamic Modeling with QUAKE/W 2007. Calgary, Alberta, Canada.

GEO-SLOPE (2012), Stability Modeling with SLOPE/W 2012. Calgary, Alberta, Canadá.

González M A, M M Lavista, L A González y A A Hernández (2018), "Análisis tenso-deformacional no lineal dinámico de estructuras de tierra". Revista Cubana de Ingeniería, 9, 49-59

Hernández, F (2012), "Asentamientos permanentes por sismo en presas de tierra y enrocamiento". Ponencia. Repositorio Digital Institucional del Instituto Politécnico Nacional. https://www.repositoriodigital.ipn.mx/handle/123456789/5601 
Ishibashi I y X Zhang (1993), "Unified dynamic shear moduli and damping ratios of sand and clay". Soils and foundations, 33, 182-191. DOI: 10.3208/sandf1972.33.182

Janbú N (1996), Slope stability evaluations in engineering practice. ISL, Trondheim.

Malley J O (1998), SAC Steel Project: Summary of phase 1 testing investigation results. Engineering Structures, 20, 300-309. DOI: 10.1016/S0141-0296(97)00033-3

MDOC (2008), Manual de diseño de obras civiles. Diseño por sismo. En: Electricidad, C.-F. D. (ed.). México.

Mesa, M (2017), "Empleo de la modelación para el diseño de terraplenes altos de carretera". Tesis presentada en opción al grado científico de doctora en ciencias técnicas, Universidad Central "Marta Abreu" de las Villas.

Mesa, M y J Álvarez (2011), "Calibración numérica de un problema de ingeniería vial". Revista de la Construcción, 10 3, 52 - 63. DOI: 10.4067/S0718-915X2011000300006

Mesa, M, J Álvarez, E Tejeda y C Recarey (2016), "Determination of the domain dimensions in embankment numerical modeling". DYNA 83 44-48. DOI: 10.15446/dyna.v83n198.49211

Mesa, M y P Guada (2013), Métodos empírico-mecanisista empleados para el cálculo del factor de seguridad en la estabilidad de taludes. Monografía. La Habana.

Mesa, M, F Lamas y E Tejeda (2014), "Estudio geotécnico y experimental de suelos para terraplenes". Memorias de la 17 Convención Científica de Ingeniería y Arquitectura,

Morgenstern N R y V E Price (1965), The analysis of the stability of general slip surfaces, Geotechnique, March. DOI: 10.1680/geot.1965.15.1.79

NC-856:2011 (2011), Carretras - Protección de taludes - Requisitos de diseño. 1 Edición,

Oñate, E (2009), Cálculo de estructuras por el método de elementos finitos, Barcelona, España, CIMNE.

Ortiz, W F (2014), "Estudio de estabilidad de los taludes de las presas del proyecto Pacalori, teniendo en cuenta la acción sísmica". Tesis de pregrado. Universidad de Cuenca. http://dspace.ucuenca.edu.ec/handle/123456789/20906

Ruesta, P F, J Díaz y J E Alva (2006), "El coeficiente sismico en el diseño de presas de tierra y de enrocado". http://www.jorgealvahurtado.com/files/redacis31_a.pdf

SAC (1999), The SAC Joint Venture [Online]. SAC Steel Project | 201 Redwood Shores Parkway, Suite 240 |Redwood City, California $94065 \mid$ (650) 595-1542. Disponible en: http://www.sacsteel.org/design/index.html

Sepúlveda, A (2011), "Comportamiento sísmico de taludes de roca. Aplicación del método de Newmark". Tesis (Master), Universidad politécnica de Madrid.

Simancas, O (1999), "Capacidad de carga de cimentaciones superficiales". Tesis doctoral, Universidad de Oviedo.

Spencer, E A (1967), "A method of analysis of the stability if embanknents assuming paralled inter-slice forces", Geotechnique. DOI: 10.1680/geot.1967.17.1.11

Suárez, J (2008), Comportamiento sísmico de los taludes. En: Suárez, J Deslizamientos: Tomo I: Análisis geotécnico. Capítulo 7. https://www.erosion.com.co/deslizamientos-tomo-i-analisis-geotecnico.html

Talukdar, P, R Bora y A Dey (2016), "Stability analysis of ash dykes in static, pseudostatic and seismic conditions". 1st International Conference on Civil Engineering for Sustainable Development Opportunities and Challenges (CESDOC) at: Guwahati, India

Torres, J A (1986), La estructura de la explanación. En: Matamoros, L. J. L. L. (ed.) Diseño y Construcción de Explanaciones. La Habana, Cuba: Avenida del Boque.

Zenghui,Z, W Weiming y Y Jixing (2014), "Quantitative analysis of parameters' influence on the stability of coal roadway clamped by upper and lower soft rock with extra thickness". International Journal of Modeling, Simulation, and Scientific Computing, 5, 15. DOI: 10.1142/S1793962313500232

Zienkiewicz, O C y R L Taylor (2002), Tensión y deformaciones planas. En: El método de los elementos finitos. 4 ed. Barcelona, España: CIMNE. Norte, M. J. (ed.) 